\title{
Los tres Méxicos: análisis de la distribución espacial del empleo en la industria y los servicios superiores, por tamańo urbano y por región
}

\author{
ISABEL ANGOA**, SALVADOR PÉREZ-MENDOZA*** Y MARIO POLĖSE**** \\ ** Profesora Benemérita Universidad Autónoma de Puebla, Facultad de Economía, BUAP. \\ *** Profesor Benemérito Universidad Autónoma de Puebla, Facultad de Economía, BUAP. \\ **** Profesor Institut National de la Recherche Scientifique
}

\begin{abstract}
The location of employment in manufacturing and in high-order services in Mexico is examined using location quotients. Municipalities are grouped into nine urban size classes and three regions: north, centre, and south. Location patterns are generally consistent with models in economic geography. High-order services concentrate in the largest urban areas, exhibiting a hierarchical pattern. Manufacturing, especially mid-tech manufacturing, is relatively more concentrated in medium-sized cities. Mexico also exhibits distinctive features: financial services are proportionately very concentrated in the capital; industrial deconcentration is still limited. The proximity of the US generates a distinctive spatial logic in the North, different from that in the Centre and the South.
\end{abstract}

KEYWORDS: location, manufacturing industry, high-order services, Mexico, regions

RESUMEN Se analiza la localización del empleo en la industria y los servicios superiores en México utilizando cocientes de localización. Los municipios están agrupados en nueve clases de tamaño urbano y en tres regiones: norte, centro, sur. En términos generales, se observan patrones de localización consistentes con los modelos que existen en la geografía económica. Los servicios superiores se concentran en las zonas urbanas más grandes, exhibiendo un patrón jerárquico. La industria, particularmente la de tecnología media, está relativamente más concentrada en ciudades de tamaño medio. Pero México muestra también ciertas particularidades: los servicios financieros están proporcionalmente muy concentrados en la capital; la desconcentración de la industria es aún limitada. La proximidad con los Estados Unidos genera una lógica espacial particular en el norte, diferente de la del centro y del sur.

PALABRAS CLAVE: localización, industria manufacturera, servicios superiores, México, regiones.

Recibido el 21 de octubre de 2007, aprobado el 17 de noviembre de 2008.

Correspondencia: Isabel Angoa y Salvador Pérez-Mendoza, Av. San Claudio y 22 sur S/N, 72570 San Manuel, Ciudad Universitaria, Puebla, Puebla, México. E-mail: iangoa@eco.buap.mx, salpere@siu.buap.mx. Mario Polèse, INRS Urbanisation, Culture et Société 385 Sherbrooke E., Montreal, QC, H2X 1E3, Canadá. E-mail: mario. polese@ucs.inrs.ca 


\section{Introducción}

El objetivo de este artículo es analizar la localización del empleo manufacturero y de los servicios superiores en México, mediante el uso de cocientes de localización. El estudio se realiza atendiendo dos criterios: la región de pertenencia (centro, norte, sur) y el tamaño urbano. Para esto, se agrupa a los 2.449 municipios de México en nueve categorías de tamaño urbano. A su vez, se definen diez clases manufactureras y doce de servicios superiores. Para estos últimos, la literatura da cuenta de múltiples denominaciones para referir a la misma realidad: servicios a las empresas, servicios a la producción, servicios intermediarios, servicios modernos (Stanback, 1984; Daniels, 1985; Moreno y Ecolano, 1992; Sassen, 2001). Se trata de servicios que pueden, en muchos casos, intercambiarse a través de grandes distancias, como los bienes fabricados. Los servicios financieros son un buen ejemplo, pues no es necesario vivir en la Ciudad de México para comprar acciones de la Bolsa de Valores de esta ciudad. La publicidad es otro ejemplo; aunque concebida en la Ciudad de México, puede ser difundida al resto del país (e incluso en el mundo), por medio de la televisión, la radio e Internet.

En el curso de los últimos ańos, se ha acumulado una abundante literatura empírica que analiza la localización de las actividades económicas en el espacio nacional (Alonso et al., 2004; Desmet y Fafchamps, 2005; Gaigné et al., 2005; Henderson, 1997, 2003; Ingram, 1998; Polèse y Shearmur, 2004, 2006; Rubiera, 2005; Terral y Polèse, 2006). Estos estudios han abordado el comportamiento de la industria y los servicios en países industrializados ${ }^{1}$ (destacan los casos de España, Francia, Estados Unidos y Canadá). A pesar de las diferencias geográficas e históricas entre países, en general, se observan las mismas grandes tendencias (Henderson et al., 2001), esto es, que los servicios al productor se concentran en las ciudades más grandes, mientras que la manufactura lo hace en ciudades de tamaño medio.

La pregunta que da base al artículo es si en México se observan las grandes tendencias de localización antes mencionadas, tomando en consideración la conjunción de tres rasgos fundamentales: i) no es aún un país plenamente industrializado; ii) se caracteriza por fuertes desigualdades regionales (no es exagerado hablar de un México dividido en tres) (Delgadillo et al., 2001; García-Verdi, 2005; Rodríguez-Pose, 2005); iii) es un país en desarrollo con una frontera común con uno de los países más ricos (Calderón y Martínez, 2004). Es razonable pensar que estos tres rasgos tendrán un impacto en el patrón de localización de las actividades económicas. Por ejemplo, es posible que el empleo manufacturero en México esté aún relativamente muy concentrado en las ciudades grandes, lo que contrasta con lo que sucede en Canadá o Estados Unidos (Terral y Polèse, 2006).

\section{Concentración de los servicios superiores y desconcentración industrial}

La literatura internacional hace referencia a dos grandes tendencias que se desprenden de estudios empíricos para países industrializados. En lo que se refiere a la industria manufacturera,

\footnotetext{
En este artículo se considera como sinónimos países industrializados o desarrollados. De antemano, definimos el desarrollo como un proceso determinado por el aumento sostenido y permanente del ingreso real per cápita, con repercusión en la calidad de vida de la población.
} 
se observa un movimiento de desconcentración a costa de las ciudades más grandes y en beneficio de las ciudades medianas y pequeñas. Es el caso de las industrias que utilizan menos materia gris, a las que podemos llamar industrias de baja y mediana tecnología. Dos ejemplos típicos son la industria automotriz y la electrónica, particularmente la fabricación de aparatos para el hogar y otros productos estándar como radio receptores, televisores, entre otros. En contraparte, se observa también una fuerte concentración en las ciudades más grandes, de lo que puede llamarse terciario superior, una relación que parece seguir siendo estable, a pesar de la introducción del Internet y otras tecnologías de la información (Gaspar y Glaeser, 1998; Sassen, 1991; Klaus y Fafchamps, 2005). Polèse y Shearmur (2006) observan, por ejemplo, el caso de Canadá, donde el nivel de concentración de sectores como el de finanzas, publicidad y otros servicios a las empresas, no ha cambiado casi nada en el curso de treinta ańos. Los servicios superiores siguen siendo sensibles al tamańo urbano (Sobrino, 2003, p. 91).

La desconcentración de la industria manufacturera, por una parte, y la concentración de los servicios superiores, por otra, no son hechos independientes. Ambos están estrechamente relacionados. En la literatura inglesa se habla de un fenómeno de crowding-out (Ingram, 1998; Graham y Spence, 1997), que puede traducirse como "expulsión industrial”. Los fundamentos económicos de este proceso han sido bien explicados por Henderson (1997). En términos simples, la concentración de los servicios superiores -el sector más dinámico de las economías modernas- en las grandes ciudades hace aumentar los precios del suelo y los salarios, acentuando también los problemas de congestión vial. Por lo tanto, las industrias que no pueden asumir los precios inmobiliarios elevados ni los altos salarios, y que son sensibles a la congestión vial, van en busca de otra localización (Henderson, 1997). Se trata generalmente de industrias de bajo (o mediano) contenido tecnológico, que en México son importantes por la gran cantidad de empleos industriales ${ }^{2}$ que generan. Estas industrias suelen ser importantes consumidoras de espacio, y por lo tanto, sumamente sensibles a los precios del suelo. Así, es en la periferia de las grandes aglomeraciones y en las ciudades medias donde se encuentran las grandes plantas de fabricación de automóviles, de electrónica y de otros equipamientos pesados. Paralelamente, si los costos suben demasiado en la parte "central" del país, donde se concentran las ciudades más grandes, las industrias más sensibles a los salarios tenderán a elegir sitios en la periferia, donde el costo de la fuerza de trabajo es menor (Henderson et al., 2001). Este movimiento de dispersión industrial se observa, por ejemplo, en Estados Unidos, donde la industria del vestido (inicialmente concentrada en la región de Nueva York) y la industria textil (al principio concentrada en Nueva Inglaterra), con el tiempo se han desplazado hacia los estados del sur, los más pobres de ese país.

La literatura internacional subraya igualmente la importancia de las economías de aglomeración: las ventajas económicas de la concentración (Fujita y Thisse, 2002). La distinción más importante se encuentra entre las economías de urbanización-las ventajas generales de la gran ciudad- y las economías de localización -relacionadas con los reagrupamientos de industrias o de actividades conexas en una misma ciudad o región-. Se emplea con frecuencia

2 Según nuestro propio sistema de clasificación, las industrias de alta tecnología participan con 5\% del empleo total en 2003. 
el término "cluster" para referir a tales agrupamientos (Porter, 1998, 2000). Las economías de localización, propias de los clusters, se asocian con más frecuencia a la industria manufacturera, y se basan principalmente en la presencia de una oferta de mano de obra especializada, de infraestructura y del establecimiento de relaciones interindustriales (Henderson, 2003). Estos clusters industriales, dependiendo de sus atributos, no se encuentran forzosamente en las ciudades más grandes.

El principal motor de la desconcentración industrial sigue siendo el crecimiento de los servicios superiores -finanzas, gestión, servicios técnicos y científicos, medios, etc.-, sensibles a las economías de localización y de urbanización. Su propensión a concentrarse en las ciudades más grandes descansa en gran parte en la necesidad continua de los contactos cara a cara. La tendencia de los grandes bancos e instituciones financieras a agruparse a menudo en un mismo barrio es una ilustración elocuente. En México, el sector de las finanzas está aún subdesarrollado, comparativamente a países más avanzados. Por lo tanto, podemos prever un crecimiento rápido de este sector, así como para otros servicios superiores, crecimiento que se hará sentir primero en el Distrito Federal así como en otras grandes ciudades como Monterrey y Guadalajara. Según la experiencia de países más industrializados, este crecimiento de los servicios superiores en las ciudades más grandes (con el consiguiente efecto de alza en los costos) expulsará consecuentemente las actividades manufactureras hacia ciudades más pequeñas.

Retomando la pregunta de partida, nos planteamos las siguientes interrogantes: ¿se concentra la industria manufacturera en las ciudades medias? ¿Se localizan las industrias tradicionales de bajos salarios en las regiones más pobres del país? ¿Se localizan los servicios superiores únicamente en las grandes ciudades? Antes de responder, es necesario observar algunas características de la geografía económica de México.

\section{México: la división del territorio en tres partes}

Se ha afirmado que la geografía económica de México se caracteriza por un rasgo particular: una larga frontera común con los Estados Unidos, que a la vez, es su principal socio comercial y fuente fundamental de inversiones extranjeras directas. El Tratado de Libre Comercio (TLC), firmado en 1994, no ha hecho más que acelerar la integración de la economía mexicana a la de su vecino del norte. $\mathrm{Al}$ respecto, ha surgido una importante literatura cuyo objetivo es analizar los efectos del TLC en el desarrollo económico regional de México (Banco Mundial, 2003; Chamboux-Leroux, 2001; Corona, 2003; Messmacher, 2000; Ornelas, 2004; Rodríguez-Pose, 2005). No es el propósito retomar este debate, sino mostrar cómo la geografía económica particular de México -cuyos orígenes se remontan mucho antes del TLC- afecta la lógica espacial de las actividades económicas.

Una primera respuesta se encuentra en el mapa 1, que ilustra los cocientes de localización del empleo manufacturero, por municipio, en el ańo 2000. Destaca a simple vista la existencia de dos Méxicos industriales que abarcan, cada uno, una porción distinta del territorio. Una primera concentración del empleo manufacturero se sitúa, grosso modo, en el eje oeste-este, que corre de Guadalajara hasta la ciudad de Tehuacán, en el Estado de Puebla, pasando por centros industriales como León, Querétaro y por la Zona Metropolitana de la Ciudad de México 


\section{Mapa 1}

COCIENTES DE LOCALIZACIÓN DE LA INDUSTRIA MANUFACTURERA EN LAS TRES REGIONES

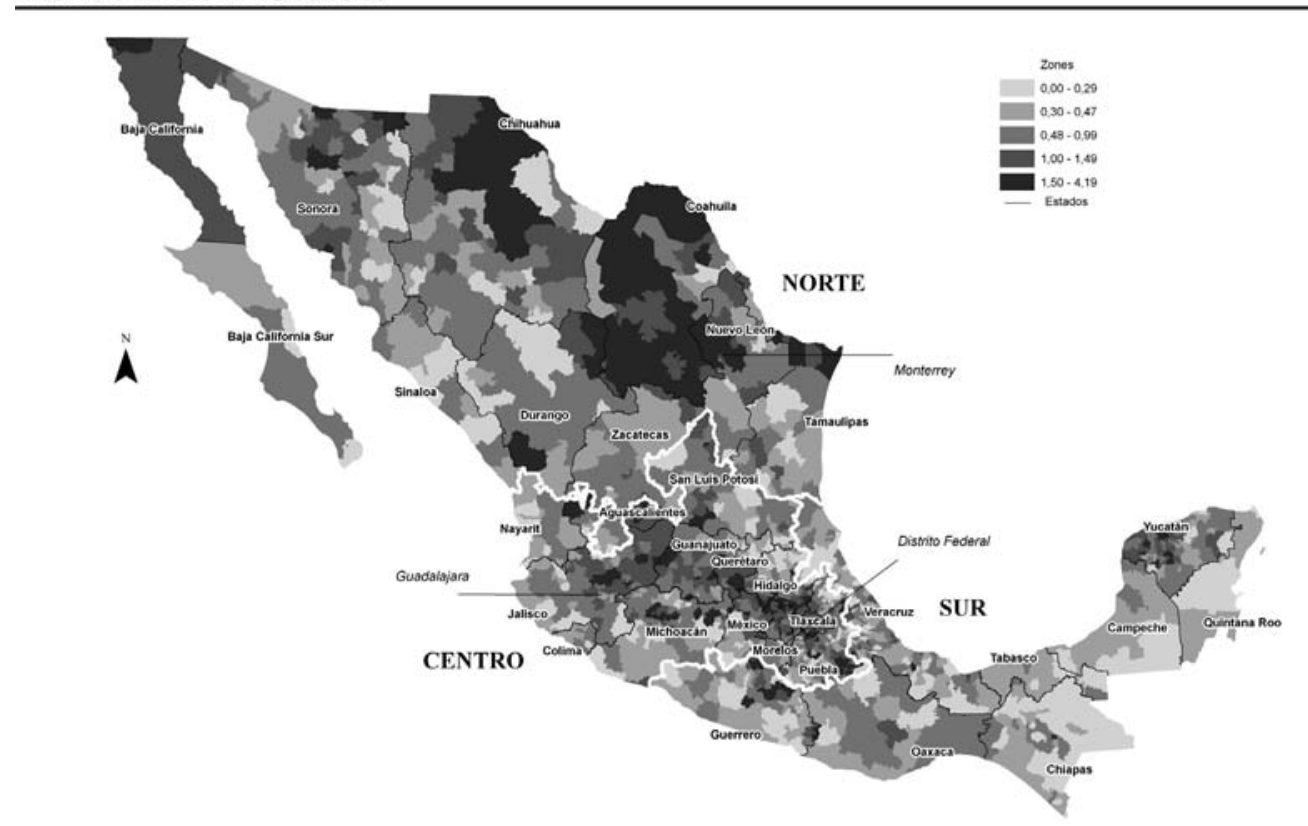

Fuente: Elaboración propia en base a datos de los Censos Económicos, 1999, INEGI, México

$(\mathrm{ZMCM})^{3}$. Este es el núcleo histórico del país, el centro. También se observa claramente una segunda zona, en apariencia más vasta (a causa sobre todo de su escasa densidad) en la parte norte del país, cerca de la frontera con los Estados Unidos.

Considerando estos aspectos, y retomando el objetivo de nuestro análisis, se ha realizado una división de México en tres grandes regiones. Por razones de simplificación, la división se ha efectuado sobre la base de las fronteras de los estados. La delimitación de las regiones centro y norte se basa en consideraciones de índole económica, y aun sociales y culturales, derivadas del desarrollo de lógicas de crecimiento diferentes, ahondadas por la cercanía del norte con Estados Unidos. Por su parte, la identificación de la región "sur" se impone porque históricamente ha sido la zona con mayores índices de marginación del país, alejada de los mayores mercados y sin grandes regiones metropolitanas.

\section{Bases de datos y metodología}

El análisis de la localización de las actividades económicas en México, a un nivel geográfico y sectorial fino (más allá del análisis por entidad federativa y del que se refiere a los grandes

\footnotetext{
3 A partir de esta línea, referirse a la Zona Metropolitana de la Ciudad de México, será estrictamente lo mismo que hablar de la Ciudad de México, pues esta última no sólo se conforma por el Distrito Federal, sino por algunos municipios del Estado de México.
} 
agregados -primario, secundario y terciario-), plantea algunos problemas. En principio, no existe una base de datos completa que contenga el conjunto de empleos de todos los sectores de la economía mexicana. Este es un elemento importante, pues en este documento se plantea analizar la localización de actividades económicas a nivel municipal, mediante la variable empleo (el dato más utilizado en la mayor parte de los estudios citados en la introducción). Sin embargo, los Censos Económicos ${ }^{4}$ que proporciona el Instituto Nacional de Estadística, Geografía e Informática (INEGI), no cubren más que ciertas ramas de la economía mexicana. Aun reagrupando todos los censos (industrial, comercial y de servicios) para un año específico, no se alcanza el 100\% del empleo del país, debido a que varios sectores no están incluidos en los datos publicados: sector primario, salud, educación, administración pública, etc. Es una limitante importante si se aplica el cociente de localización. Éste mide la concentración relativa del empleo (en el sector $x$ en la región $x$ ) en comparación con el conjunto nacional. Si, por ejemplo, la región $x$ contase con $10 \%$ del empleo nacional total real y con $15 \%$ del empleo (nacional) de textil, eso daría un cociente de 1.5 Pero, si el empleo total se subvalora-si se tiene una región rural, con una alta proporción de empleo agrícola (no considerado por los Censos Económicos)-, esto conduciría a una sobre-estimación del cociente real. Si-de acuerdo con los Censos Económicos- el empleo "total” de la región $x$ fuese únicamente $5 \%$ del "total” nacional, eso hubiera producido un cociente de 3.0, lo que daría la impresión de que la economía de la región $x$ se especializa mucho más en la actividad textil de lo que es en realidad.

En síntesis, nos falta un punto de referencia válido para evaluar el empleo total en la ecuación del cociente. Hubiera sido posible utilizar el total de los Censos Económicos (industrial, comercial y de servicios), pero con el riesgo -como acabamos de explicar- de sobrestimar sistemáticamente los cocientes (para la industria y los servicios superiores) de las regiones y municipios con una fuerte proporción de empleo en el sector agropecuario o en el público, es decir, en los municipios de menor tamaño. Debido a que el objetivo es comparar regiones y zonas urbanas de diferentes tamaños, esta opción parece problemática.

Entonces, ¿cómo resolver este problema para el que no existe solución perfecta en el caso mexicano? Se procedió de la siguiente manera. Tomamos como referencia-el denominador en el cálculo del cociente- la Población Activa Ocupada ${ }^{5}$, tal como la publica el Censo General de Población y Vivienda del 2000, comparándola -el numerador-con los datos de empleo ${ }^{6}$ del Censo Económico por sector de actividad y por región urbana. Es una solución imperfecta, porque la comparación de datos de dos fuentes distintas genera riesgos; tanto más cuanto que los Censos no miden exactamente la misma población. El Censo Económico no cubre las localidades rurales de menos de 2.500 habitantes, a menos que se trate de cabeceras municipales. No es posible estimar, de forma sistemática, el tipo de sesgo que esto introduce en

\footnotetext{
Para una explicación más detallada, revisar la dirección electrónica que ofrece INEGI: http://www.inegi.gob. $\mathrm{mx} /$ est/contenidos/espanol/metodologias/censos/metodo_ce2004.pdf

5 El INEGI considera a la población activa ocupada como la población de 12 años y más que realizó alguna actividad económica (agricultura, industria, comercio y servicios), al menos una hora en la semana de referencia, a cambio de un sueldo, salario, jornal u otro tipo de pago.

$6 \quad$ El personal ocupado representa a todas aquellas personas (hombres y mujeres) que dependen de la unidad económica y trabajaron bajo su dirección y control, cubriendo como mínimo una tercera parte de la jornada laboral, recibiendo regularmente un pago e incluso sin recibirlo.
} 
nuestros resultados. Es probable que induzca a aumentar los cocientes en las regiones más rurales, pero mucho menos que si hubiéramos utilizado el total de los Censos Económicos. Este procedimiento afectaría únicamente los resultados para las ciudades ${ }^{7}$ pequeńas clasificadas por tamaño urbano (menos de 15.000 habitantes).

Con el objetivo de evaluar los sesgos que implican los dos puntos de referencia, hemos calculado todos los cocientes: i) con el total de los Censos Económicos en el denominador; ii) con el total de la Población Activa Ocupada, también en el denominador. La comparación confirma, efectivamente, que los cocientes para la industria y los servicios superiores son sistemáticamente más altos para el sur del país y para las zonas urbanas de menor tamaño, utilizando el primer cálculo. Además, la comparación con ambas fuentes no muestra diferencias significativas en las posiciones relativas de las observaciones (zonas urbanas y municipios), lo que nos lleva pensar que los resultados presentados -utilizando la Población Activa Ocupada en los denominadores- son una representación aceptable de la realidad. El cálculo del cociente de localización, utilizando los datos provenientes de las dos fuentes, nos permite, en cierta forma, presentar comparaciones que de otra manera no habrían sido posibles, aunque es un hecho que la comparación directa de los datos provenientes de los dos censos no permite llegar a porcentajes reales (digamos, el porcentaje del empleo en la industria textil en el empleo total de la región $x$ ). Sin embargo, la interpretación de los cocientes no plantea problemas conceptuales mayores. Puesto que las posiciones relativas de las observaciones -regiones o zonas urbanas- muestran el mismo patrón en casi todos los casos, poco importa el método de cálculo utilizado (aunque tomando en cuenta el sesgo descrito en los párrafos anteriores). Por lo tanto, se piensa que la comparación de dos ańos diferentes $(2000,2003)$ no genera mayor sesgo. Todas las figuras que se presentan en este estudio surgen del mismo procedimiento y, por lo tanto, de la misma metodología de base.

\section{La clasificación de las observaciones (regiones urbanas y municipios)}

Para definir el sistema urbano mexicano, se trabajó con datos proporcionados en las publicaciones $^{8}$ del INEGI hasta el último nivel geográfico, los municipios. Hasta el año 2000, se consideraban 2.449 municipios. Estos se agruparon en tres subconjuntos: centro, norte y sur de México, tomando como referencia geográfica los límites de los estados. Asimismo, se procedió a clasificarlos por tamaño urbano, considerando el número de habitantes. Con base en este criterio, se retuvieron tres zonas metropolitanas (México, Guadalajara y Monterrey), mientras que los municipios restantes fueron organizados en aglomeraciones, siguiendo la metodología propuesta por el Consejo Nacional de Población para, finalmente, organizar el

\footnotetext{
En este documento nos referimos a ciudad como sinónimo de municipio. Es claro que una ciudad puede rebasar la unidad geográfico-administrativa, o sea el municipio. Sin embargo, las fuentes y el nivel de desagregación de los datos utilizados en este artículo sólo permiten el tratamiento de esta información a este nivel geográfico.

8 El INEGI posee seis Censos Económicos que se ofrecen en forma impresa y medios electrónicos, comenzando por el de 1981. Es posible mencionar que la metodología ha ido mejorando con el tiempo, puesto que, en los últimos dos Censos (1999 y 2004), la clasificación, antes sólo para México, se aplica ahora considerando a los países asociados al TLC.

9 Se consideraron las aglomeraciones por distancias en los estados y en algunos casos las que forman ciudades de diferentes estados. No es el objetivo, en este artículo, trabajar este análisis, lo destacamos con el objetivo de que
} 
sistema urbano por tamaño de población: de 1 a 2 millones, de 500 mil a 1 millón, de 100 mil a 500 mil, de 50 mil a 100 mil, de 15 mil a 50 mil y de menos de 15 mil.

\section{La clasificación de las variables (los sectores de actividad económica)}

Para el análisis de la industria y de los servicios superiores, se utilizó la información del XIII Censo Industrial, Comercial y de Servicios, y del XVI Censo Industrial que proporciona el INEGI, con datos a nivel municipal y para las delegaciones del Distrito Federal, atendiendo a la característica censal denominada "personal ocupado".

El uso del Sistema de Clasificación Industrial de América del norte, SCIAN ${ }^{10}$, indujo a trabajar, para el caso de los servicios superiores, con un mayor nivel de desagregación, por lo que se analizaron 45 ramas de actividad económica, integradas en 12 grupos: impresión y edición, espectáculos y producción artística, legales y de contabilidad, ingeniería, ciencia y diseño, consultoría en computación, gestión y administración, publicidad, otros servicios a empresas, financieros y de seguros, inmobiliarios y de alquiler.

La actividad industrial, por su parte, siguió idéntico proceso de reagrupamiento de las ramas de actividad en diez sectores de análisis: la industria tradicional, que incluye ramas que no exigen un contenido alto en tecnología y son utilizadoras intensivas de mano de obra, no necesariamente de alta calificación; la industria textil y la del vestido, analizadas de forma separada por las características de cada una en cuanto a localización y uso de mano de obra; otras industrias modernas, en las que se incluyeron las ramas como automotriz, eléctricos y del hogar, entre otros; industria de alta tecnología, la cual incluye ramas como la farmacéutica, producción de equipo informático y altamente especializado y aeronáutico, que recientemente se agrega a los Censos Económicos.

\section{Resultados}

Se comienza por los resultados de las tres grandes regiones (tres agregados), para posteriormente, en un intento por tejer más fino, presentar los relativos al tamaño urbano en cada región.

se tome en cuenta que los tamaños urbanos contienen las aglomeraciones reconocidas oficialmente.

10 El Sistema de Clasificación Industrial de América del norte (SCIAN), fue elaborado, acordado y firmado por el INEGI, Statistics Canada y The Office of Management and Budget de Estados Unidos. este nuevo sistema sustituye la clasificación de actividades económicas utilizadas en México, en Estados Unidos y en Canadá para la generación de su estadística económica. En México se utilizó por primera vez en los Censos Económicos de 1999. 


\section{Figura 1}

\section{CONCENTRACIÓN RELATIVA DEL EMPLEO EN DOS GRANDES SECTORES \\ TRES REGIONES (México $=1.00$ )}



Fuente: Elaboración propia con base en datos del XVI Censo industrial y del XIII Censo de servicios, INEGI

\section{Por Región}

La Figura 1 proporciona los cocientes de localización entre el empleo total para los dos grandes sectores económicos y para las tres regiones en que se dividió México. La mirada a los "tres Méxicos" pone de manifiesto la marginación del sur. Tanto para los servicios superiores como para la industria manufacturera, el sur se encuentra totalmente por debajo del promedio nacional.

La diferencia entre el centro y el norte es menos marcada, pero no por ello menos clara. El norte está más industrializado que las otras dos partes del país, otro indicador de la importancia de la frontera norte y, paralelamente, del acceso al mercado americano como factor de localización (Castillo et al., 2004). Por su parte, la sobrerrepresentación -aunque ligera- del centro en el plan de los servicios superiores, refleja el rol dominante de la Zona Metropolitana de la Ciudad de México como centro de servicios para el conjunto del país.

La Figura 2 muestra resultados desagregados para los diez sectores manufactureros, clasificados (de izquierda a derecha) siguiendo, grosso modo, su nivel de contenido tecnológico ${ }^{11}$. La vocación industrial del norte se encuentra sobre todo en los grandes sectores, entre los que destacan: material de transporte (automóviles, camiones, materiales ferroviarios, entre otros) y electrónica (aparatos para el hogar, equipos de audio, TV, radios, etc.). La imagen es, por lo tanto, muy distinta de lo que se ve habitualmente en una región dominada por las maquilas tradicionales

11 Esta clasificación es de los autores, según su criterio; no debe atribuírsele una precisión demasiado importante. 
de la industria del vestido y textil ${ }^{12}$. Al parecer, el norte vive una evolución similar a la de los nuevos países industrializados (Corea del sur y Taiwán), que han pasado progresivamente del vestido a la electrónica, y ahora a sectores cada vez más complejos. Paralelamente, este patrón es igualmente coherente con un movimiento de desconcentración industrial estimulado por salarios más elevados en las grandes ciudades. Es probable que varias industrias tributarias de mano de obra barata, principalmente vestido y textil, estén abandonando el norte, en busca de una localización menos cara al sur de México.

\section{Figura 2}

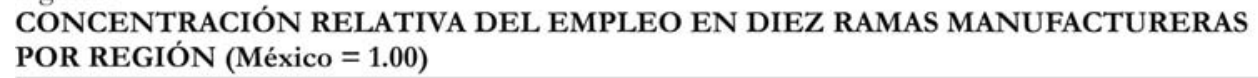

POR REGIÓN (México $=1.00)$

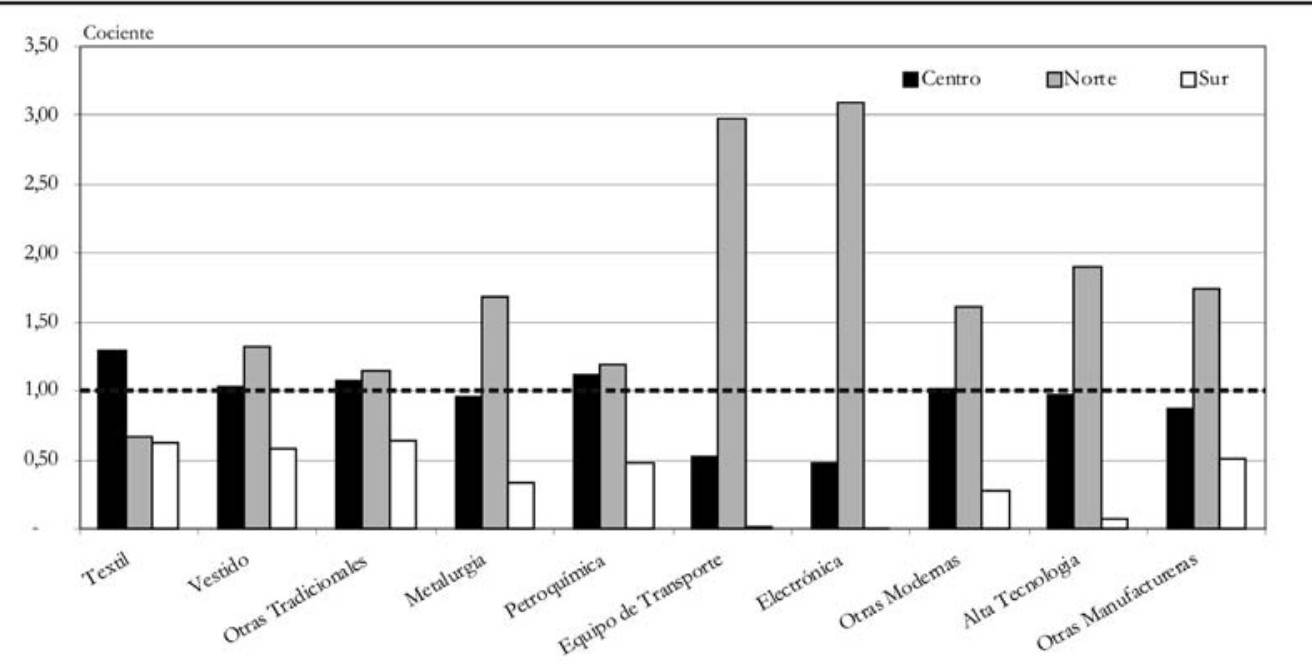

Fuente: Elaboración propia con base en datos del XVI Censo industrial, INEGI

Como se ilustra en la Figura 2, los pocos empleos industriales que se encuentran en el sur están proporcionalmente concentrados sobre todo en la industria del vestido, de los textiles y otros sectores más tradicionales (fabricación de alimentos y bebidas) ${ }^{13}$. Pero, en todos los casos (incluyendo los sectores más tradicionales), la parte del sur sigue estando sensiblemente por debajo del promedio nacional. Excepto en el caso de los textiles, fuertemente concentrados en el centro, el norte acapara una parte más que proporcional de las industrias "tradicionales", aunque su fuerza radica cada vez más en las industrias innovadoras.

El centro posee, dentro del conjunto, una estructura manufacturera más diversificada, con una presencia relativamente fuerte en todos los sectores, salvo en los considerados "faros" del norte; lo que permite pensar que su producción industrial está más orientada hacia el mercado interno.

12 De hecho, el empleo combinado en el norte en 2003 en la electrónica es cuatro veces superior al total para el textil y el vestido.

13 Los tres primeros sectores en la Figura 2, cuentan, aproximadamente, por tres cuartas partes (75\%) de todo el empleo manufacturero en el sur, contabilizados en el Censo Económico 2003. 
Las diferencias para los servicios superiores (Figura 3) son menos claras a primera vista, aunque no menos reveladoras. La fuerza relativa del centro radica, en primer lugar, en la publicidad, los servicios informáticos y financieros, lo que refleja seguramente el rol dominante de la Zona Metropolitana de la Ciudad de México como centro nacional. Incluso si el norte posee en ciertos aspectos una estructura industrial más moderna que el centro, esto no parece traducirse en una vocación dominante (nacional) en cuanto a los servicios superiores. Los tres servicios para los que el norte muestra los valores -cocientes- más elevados son, respectivamente, los "otros servicios" (rubro en el que entra todo lo que no es clasificable), alquiler y servicios inmobiliarios, que no son servicios de vocación nacional.

\section{Figura 3}

\section{CONCENTRACIÓN RELATIVA DEL EMPLEO EN DOCE RAMAS DE SERVICIOS}

POR REGIÓN $($ México $=1.00)$

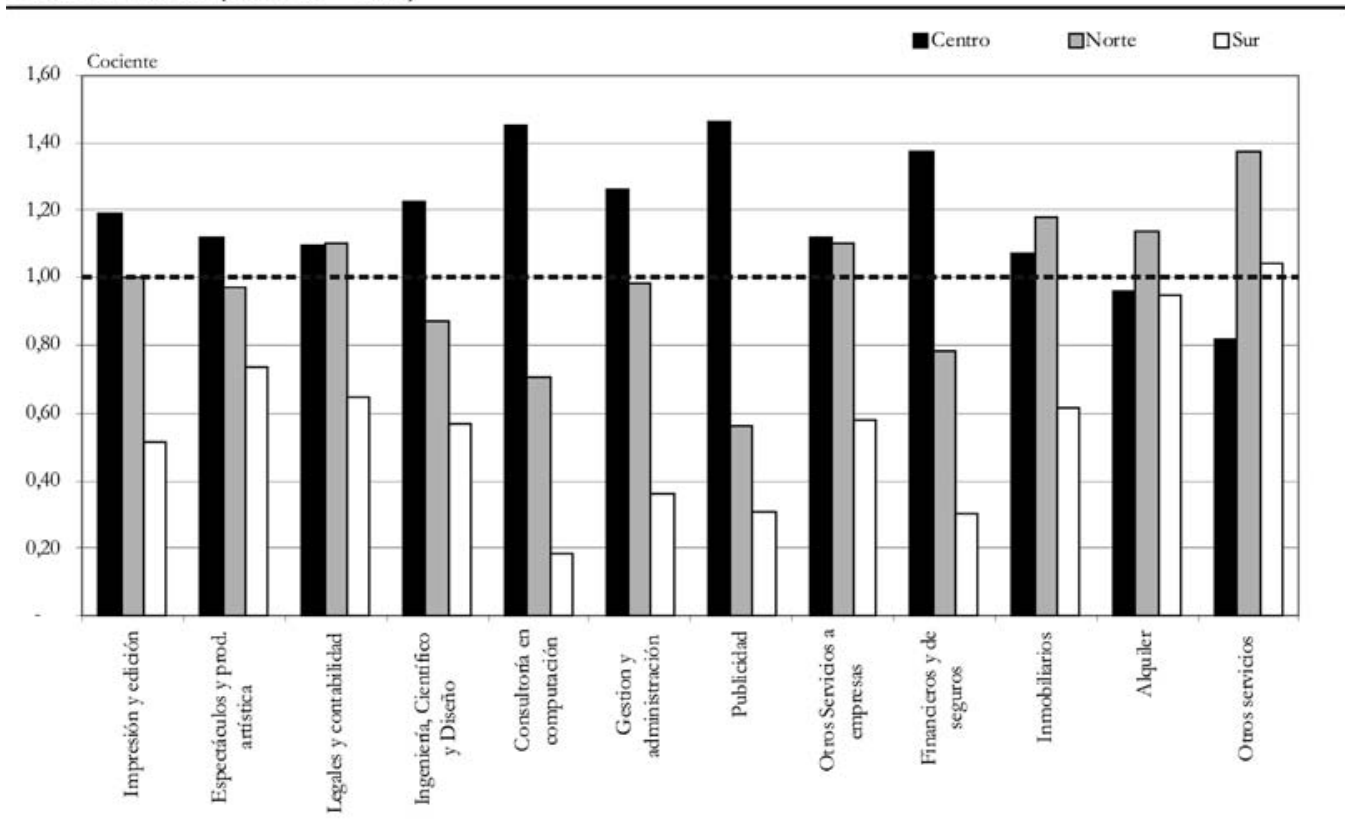

Fuente: Elaboración propia con base en datos del XIII Censo de servicios y del XVI Censo Industrial, INEGI

Diversas explicaciones son posibles. En primer lugar, puesto que las industrias manufactureras del norte son a menudo filiales extranjeras situadas a proximidad de la frontera, es posible que una parte de los servicios (financieros, informáticos, etc.) que les son necesarios sean proporcionados por la casa matriz o empresas de consultores del otro lado de la frontera, en San Antonio, El Paso, Los Ángeles, u otras ciudades americanas próximas. En segundo lugar, es probable que buena parte de las actividades de fabricación en el norte incluyan principalmente actividades de ensamblado que no necesitan un aporte importante en servicios exteriores ${ }^{14}$. Por ejemplo, basta que un programador pase de vez en cuando (quizá,

14 La forma de recolección de los datos por parte de las agencias estadísticas nacionales no permite distinguir entre 
incluso, de los Estados Unidos) para reprogramar las máquinas para que la fabrica funcione. Finalmente, la geografía desempeña sin duda un papel importante. Varias ciudades grandes del norte -pensemos en Juárez o Tijuana- están situadas demasiado lejos del núcleo del mercado mexicano para poder aspirar a una vocación nacional como centros de servicios. Sin duda, la mejor explicación radica en la combinación de estos factores.

De nueva cuenta, el sur se muestra como el pariente pobre. No sólo porque sus cocientes se encuentran sistemáticamente por debajo del promedio nacional (salvo en el caso de los "otros servicios"), sino porque los servicios para los que el sur muestra los mejores resultados son los dirigidos -al menos en parte- a los particulares y a los mercados locales: servicios jurídicos (y contables), alquiler, servicios inmobiliarios, artes y espectáculos. Se adivina que estos servicios son tributarios del turismo, principalmente los últimos tres. El bajo cociente del sur en servicios informáticos -sector faro de la economía del saber- es quizá el índice más problemático de las deficiencias estructurales de la economía de esta parte del país. El bajo cociente para los servicios financieros refuerza esta percepción. ¿Cómo atraer (o desarrollar) industrias modernas en el sur en ausencia de un sector informático e instituciones financieras locales fuertes? Existe una relación entre estas deficiencias y la ausencia de grandes ciudades en el sur, lo que se explorará en la siguiente sección.

\section{Figura 4}

\section{CONCENTRACIÓN RELATIVA DEL EMPLEO EN LOS SERVICIOS SUPERIORES POR REGIÓN Y TAMAÑO URBANO (México $=1.00$ )}

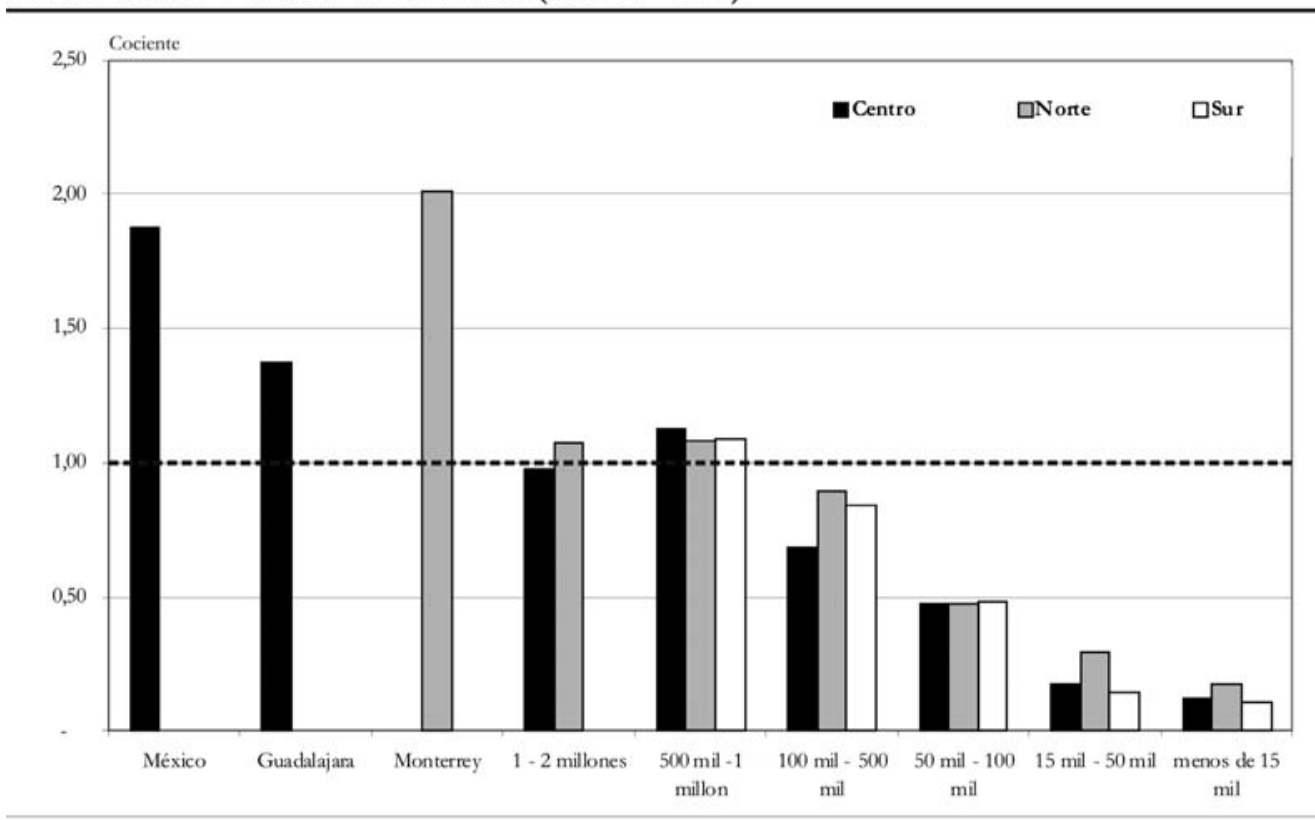

Fuente: Elaboración propia con base en datos del XIII Censo de servicios del XVI Censo industrial, INEGI

diferentes funciones al interior de un mismo sector económico. No sabemos si se trata de empleos de concepción, de diseño, de gestión, de fabricación (en el sentido propio) o de ensamblado de piezas. 


\section{Tamaño urbano}

La Figura 4 proporciona resultados para los servicios superiores (en conjunto) por región y por tamaño urbano. La imagen que se obtiene corresponde a lo esperado ${ }^{15}$. Los servicios superiores adoptan, grosso modo, un modelo jerárquico, en función del tamaño urbano. Si la distribución en la Figura 4 no parece ser perfectamente jerárquica, es porque se ha dividido a México en tres y se ha separado a las tres grandes metrópolis del país. Sin estas distinciones -sobre todo en lo alto de la jerarquía urbana- la progresión sería casi lineal, como puede observarse por debajo de la clasificación urbana de 1 a 2 millones de habitantes.

Esto nos permite dar una respuesta a la pregunta planteada al final de la sección anterior. El problema del sur parece ser de tamaño urbano, al menos en parte. El sur no comprende ninguna gran aglomeración urbana de más de un millón de habitantes ${ }^{16}$. Sin embargo, la misma Figura 4 nos dice que la proporción de los servicios superiores en la economía local -sin llegar a este umbral de población - varía casi sistemáticamente en función del tamaño urbano, sin importar la región. Si el sur muestra un bajo cociente para los servicios superiores en su conjunto (ver la Figura 1), es, en gran parte, porque comprende ciudades más pequeñas.

Las tres grandes metrópolis mexicanas dominan claramente el paisaje nacional en cuanto a servicios superiores. Sin negar el rol central de la ZMCM -en lo alto de la jerarquía- cada una posee, al parecer, su zona de influencia propia: Guadalajara, todo el oeste, y Monterrey, el noreste del país. Ya que no existe ningún contrapeso real en el sur, es probable que la ZMCM domine, sin oposición, toda esta parte del país como centro de servicios superiores, además de su función nacional. La relativa debilidad de Guadalajara se debe sobre todo a su menor desempeño con respecto a las grandes funciones de vocación nacional -finanzas, gestión- en comparación con las otras dos metrópolis ${ }^{17}$. El elevado cociente de Monterrey, en contraparte, es el índice de su papel como metrópoli económica en varios niveles ${ }^{18}$. Esto nos obliga a matizar los comentarios anteriores sobre las deficiencias del norte en materia de servicios superiores. Así, es necesario hacer una distinción entre, por una parte, Monterrey (y la región que la rodea), dotada de una buena estructura de servicios superiores y, por otra parte, ciudades fronterizas como Tijuana, Juárez y Nogales, con estructuras más deficientes.

El análisis de la concentración del empleo en las industrias manufactureras por región y por tamaño urbano mostró resultados análogos a los de la Figura 4. En México, la industria -en términos relativos- se concentra menos en las metrópolis más grandes. La Zona Metropolitana

\footnotetext{
15 La regla general es que, a mayor tamaño poblacional, más elevada es la concentración de este tipo de actividades (Sobrino, 2003).

16 Las ciudades más grandes -Acapulco, Oaxaca, Tuxtla Gutiérrez-son relativamente modestas a la escala mexicana.

17 Para no sobrecargar demasiado el texto, no mostramos los resultados detallados por metrópolis. Los dos cocientes más fuertes para Guadalajara son los servicios informáticos y los “otros servicios” a las empresas, que comprenden también los servicios técnicos y científicos diversos. Las figuras 5 a la 8, presentadas más adelante, muestran resultados detallados para ciertos sectores.

18 Monterrey destaca para los servicios más técnicos, como Guadalajara, pero muestra además buenos resultados para los servicios financieros, de gestión y de administración.
} 
de la Ciudad de México es la que parece ejercer el más fuerte efecto de expulsión industrial, lo que no es sorprendente, debido a su inmenso tamaño. El cociente se sitúa por debajo del promedio nacional, lo que indica que la capital del país no es más, en términos relativos, una ciudad industrial. Como las otras grandes metrópolis del mundo, es, en primer lugar, una ciudad de servicios. La contraparte del bajo cociente de la metrópoli mexicana se constituye por los cocientes más elevados de las ciudades del centro con poblaciones de entre $500 \mathrm{mil} \mathrm{y}$ un millón, y entre un millón y dos millones de habitantes. Se encuentran ciudades a proximidad de la Ciudad de México como Toluca, Pachuca y Puebla, pero también ciudades más alejadas como Querétaro y León. Es posible suponer que las tres primeras son localizaciones de predilección para las industrias que buscan precios inmobiliarios más bajos (y sin duda también menores costos de mano de obra), mas sin alejarse demasiado de la capital y de los servicios que ésta ofrece.

Sin embargo, también se observa que la desconcentración -en la parte central del país- sigue siendo relativamente tímida, en comparación con lo que puede observarse en ciertos países industrializados. Como se mencionó anteriormente, según Terral y Polèse (2006), el efecto de expulsión industrial comienza a hacerse sentir a partir del umbral de los 500.000 habitantes y más en Canadá y los Estados Unidos; o sea que son las aglomeraciones urbanas con poblaciones inferiores a este umbral las que muestran los cocientes más elevados. Pero en México, los cocientes más elevados -en el centro y también en las otras dos regiones- se encuentran en las clasificaciones con poblaciones superiores a los 500.000 habitantes. En síntesis, la industria sigue prefiriendo las ciudades relativamente grandes, aún cuando sigue abandonando la ciudad más grande (y seguramente, también, en parte Guadalajara y Monterrey).

¿Por qué entonces el proceso de desconcentración industrial parece menos avanzado en México? Existen diversas razones posibles. En primer lugar, la relativa debilidad -comparada con los países industrializados- de las redes de transporte y comunicación. En segundo lugar, la disparidad entre ciudades grandes y pequeñas -en términos de calidad de servicios e infraestructuras-es probablemente mayor en México que en los países industrializados. Finalmente, los servicios superiores están más concentrados en algunas (grandes) ciudades a lo alto de la jerarquía. Por ejemplo, es poco común encontrar en México ${ }^{19}$ una sucursal bancaria que ofrezca una buena gama de servicios en una pequeña ciudad de menos de 5.000 habitantes -con excepción de los destinos turísticos- mientras que se encuentran sucursales bancarias casi en todas partes en las ciudades canadienses y norteamericanas. Estas diferencias tienen por efecto frenar el movimiento de las industrias manufactureras hacia las ciudades más pequeñas ${ }^{20}$.

19 En México cerca de 35\% de la PEA tiene acceso a los servicios financieros de la banca comercial, y al menos 25 millones de habitantes en edad productiva no cuentan con esos servicios, debido principalmente a que sus niveles de ingresos están por debajo de los ingresos de los clientes típicos que acuden a la banca, a que estas instituciones cuentan con una escasa dispersión geográfica, y al alto costo de la información disponible y su administración (Silva, 2004, p. 577).

20 En México, la importancia de los servicios a la producción aumentan en el PIB del propio sector terciario, al pasar de 30,9\% en 1960, a 52,3\% en 2003. En cuanto a la estructura del empleo, la participación se incrementa de 22,3 a 35,5\% entre 1960 y 2003 respectivamente (Garza, 2006, pp. 128-129). 
El caso del norte es más complejo. La desconcentración industrial está sin duda operándose en torno a Monterrey, por ejemplo hacia Saltillo. Sin embargo, como lo hemos visto, es necesario hacer una distinción entre Monterrey y las ciudades de la frontera. El cociente más elevado -la clasificación norte, 1 a 2 millones de habitantes- está asociado con las ciudades fronterizas (Juárez y Tijuana) que son, en términos relativos, las ciudades más industrializadas del país. Cada ciudad fronteriza industrial es, en cierta forma, el área de expulsión industrial de su vis-à-vis norteamericana. La expulsión no se hace con respecto a las grandes ciudades mexicanas, sino a las ciudades norteamericanas próximas, con la ventaja adicional (para las sedes norteamericanas) de una frontera que asegura precios inmobiliarios y salariales aún más bajos del lado mexicano. No es casualidad si las ciudades fronterizas industriales están situadas frente (o a la proximidad) a una ciudad norteamericana (San Diego, Tucson, El Paso), o a lo largo de un corredor de transporte (por ejemplo, Monterrey-San Antonio).

Visto desde esta perspectiva, la desventaja del sur es doble. No tiene ni el tamaño urbano necesario, ni una buena localización para atraer a las industrias. En este sentido, el análisis realizado sugiere que la localización -es decir, la distancia- sigue siendo un factor determinante para la industria manufacturera. Para cada clasificación de tamaño urbano (por debajo de 500 mil habitantes), el cociente en el sur es sistemáticamente más débil que en las otras dos regiones del país.

\section{Sectores elegidos}

El análisis de dos sectores tradicionales (textil y del vestido) muestra en ambos casos que las concentraciones más fuertes no se encuentran en las ciudades más grandes, aunque la Zona Metropolitana de la Ciudad de México muestra una concentración relativa ligeramente por encima del promedio nacional para el textil. Para este último sector, la localización predilecta se sitúa en las ciudades medianas de distintos tamaños -entre 50.000 y dos millones de habitantes- en la parte central del país. El buen desempeño de la clasificación urbana "1 a 2 millones", confirma la posición dominante de Puebla como centro de producción textil. No se trata únicamente de un fenómeno (reciente) de maquila, sino de una tradición industrial que se remonta a siglos atrás ${ }^{21}$. En conjunto, la imagen que se observa -considerando la posición dominante de las ciudades del centro- es la de una industria en la que gran parte de la producción está dirigida al mercado interno.

Por el contrario, se puede suponer que el elevado cociente para ciudades muy pequeñas del sur (menos de 15.000 habitantes) es el índice de firmas en busca de mano de obra (sobre todo femenina) de bajo salario. Ya hemos evocado la hipótesis de un desplazamiento hacia el sur de las industrias con salarios bajos, a medida que los salarios aumentan en las ciudades del norte.

\footnotetext{
21 En México, hacia 1845, la distribución de la industria textil se extendía en torno a 23 entidades, que poseían al menos un establecimiento. Puebla era la localidad con el mayor número de empresas, sumando 21 de un total de 99 , o sea $21,2 \%$ del total nacional (Garza, 1985, p. 97).
} 
La industria del vestido se presta más fácilmente que la industria textil a actividades de maquila ${ }^{22}$, lo que se refleja en su distribución espacial. En primer lugar, la industria del vestido está poco presente en las tres grandes metrópolis del país. Asimismo, se localiza en ciudades medianas (y pequeñas) del centro y del norte, a diferencia de la industria textil, con una presencia también bastante apreciable en ciudades medias del sur. Pero su distribución en el sur sigue siendo tímida. Excepto en el caso de las ciudades clasificadas entre 500.000 a 1 millón, los valores del sur siguen estando sistemáticamente por debajo de las otras dos regiones. Nuevamente, esta región muestra que las ventajas asociadas con un tamaño pequeño (menores costos) no son suficientes, al parecer, para anular las desventajas de la distancia de los mercados.

La industria del vestido funciona según dos lógicas espaciales diferentes. La primera (más orientada hacia los mercados internos, con las ciudades de México y Puebla como pivotes), comprende las ciudades medianas (y pequeñas) del centro, pero también las del sur. Pueden observarse, por ejemplo, importantes concentraciones de empleo en la industria del vestido en Tehuacán y Teziutlán, dos ciudades del estado de Puebla, una mediana y otra pequeña. En ambos casos, es muy probable que las telas - para su costura- lleguen de la Zona Metropolitana de la Ciudad de México o de Puebla, para ser reenviadas a estas dos ciudades ${ }^{23}$. La otra lógica espacial, orientada sobre todo a los mercados exteriores (principalmente Estados Unidos y Canadá), gira en torno a las ciudades (en su mayoría fronterizas) del norte, con los centros norteamericanos de textiles y del vestido como pivotes. Es posible suponer que los valores elevados de las ciudades más grandes del norte - excepto Monterrey- y de las ciudades pequeñas de 15.000 a 50.000 habitantes, reflejan una división entre las actividades más avanzadas tecnológicamente y las actividades que emplean más bien una mano de obra poco calificada a bajo salario.

22 La maquila exige que los insumos materiales sean importados, ensamblados y luego reexportados. En el caso de la industria del vestido, se trata de telas importadas, ensambladas (cosidas generalmente) y reexportadas.

23 En el sur, en Chiapas para ser precisos, uno de los autores visitó una fábrica de jeans, donde las telas (y también los diseños que se debían seguir) llegaban todas las semanas de Puebla. Los propietarios eran de Puebla. 


\section{Figura 5 \\ CONCENTRACIÓN RELATIVA DEL EMPLEO EN LA INDUSTRIA ELECTRÓNICA POR REGIÓN Y POR TAMAÑO URBANO (México $=1.00$ )}



Fuente: Elaboración propia con base en datos del XVI Censo industrial, INEGI

Esta doble lógica espacial funciona igualmente para la industria electrónica (Figura 5), pero con pivotes urbanos diferentes. La mayor parte de la industria electrónica está dirigida hacia el exterior, y ha reemplazado a la industria del vestido en las ciudades fronterizas más grandes como primera fuente de empleo de tipo maquila. Pero una segunda lectura de la misma figura muestra que las ciudades del centro, principalmente Guadalajara y las clasificadas entre 500.000 a 1 millón de habitantes, exponen resultados relativamente positivos. No hay que olvidar que el promedio nacional se sitúa en 1.00. La fuerte concentración relativa de los empleos en la industria electrónica en algunas ciudades fronterizas tiene como efecto (visual) "disimular" el buen desempeño de otras ciudades. Esto sugiere que se están formando clusters propiamente mexicanos especializados en industria electrónica en torno a las zonas metropolitanas de Guadalajara y Monterrey, pero que no son funciones de maquila (importación - ensamblaje - reexportación).

La posibilidad de un cluster mexicano, sin relación con la frontera, especializado en industria electrónica y otras actividades conexas (principalmente fabricación de computadoras) se manifiesta aún más claramente en el análisis de la concentración relativa del empleo en la industria de alta tecnología por región y por tamaño urbano= Nuevamente se deduce que las grandes ciudades fronterizas del norte ocupan la primera posición en los empleos de alta tecnología. Pero esta vez, la Zona Metropolitana de Guadalajara muestra un cociente bastante elevado. En este caso, es razonable pensar que son las economías de aglomeración que ofrece una gran ciudad como Guadalajara - principalmente por la presencia de una oferta de mano de obra y servicios especializados- las que prevalecen sobre la proximidad con la frontera. Esto es lo propio de un cluster industrial. La región de Guadalajara no es probablemente el único ejemplo 
entre las ciudades del centro. En contraste, es un tanto desolador observar hasta qué punto las ciudades del sur están ausentes.

\section{Figura 6 \\ CONCENTRACIÓN RELATIVA DEL EMPLEO EN SERVICIOS DE CONSULTORÍA Y COMPUTACIÓN. POR REGIÓN Y POR TAMAÑO URBANO $($ México $=1.00)$}

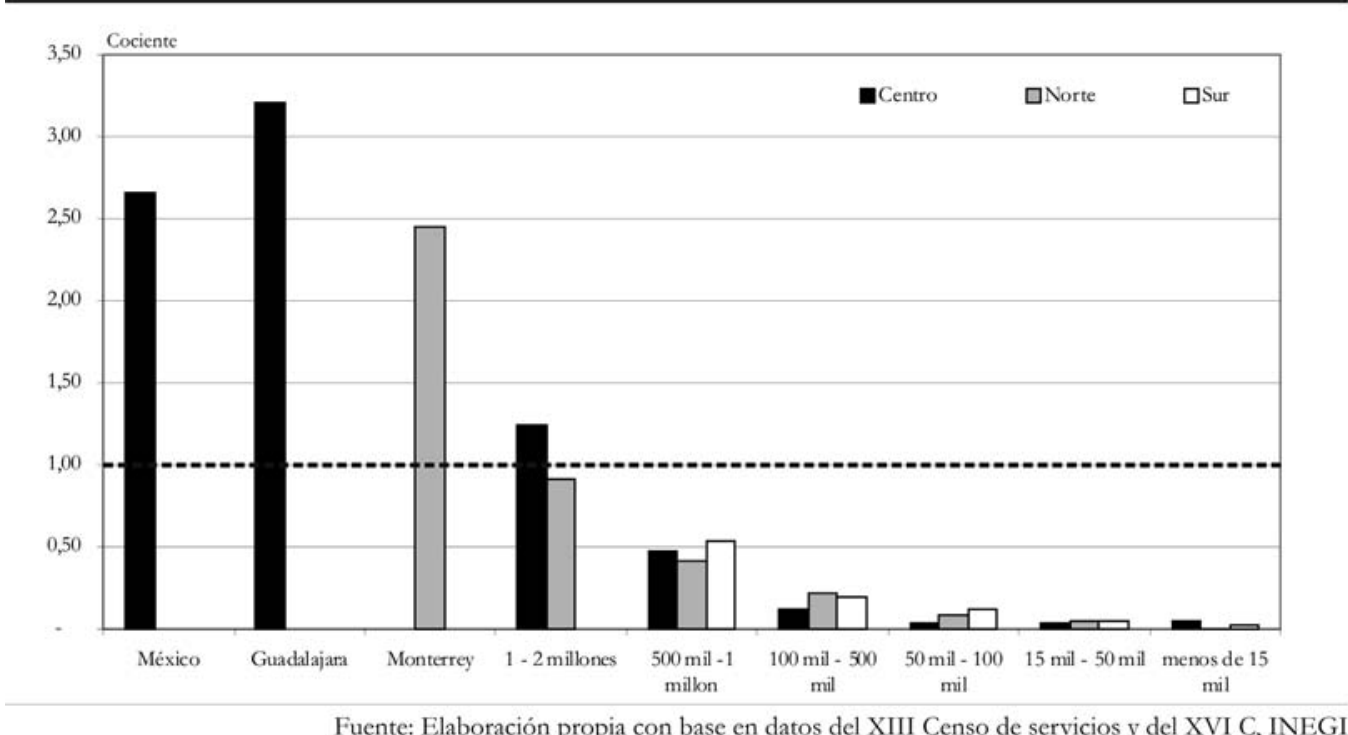

El análisis para tres sectores de servicios a las empresas plantea los siguientes puntos. Los servicios de consultoría e informáticos (Figura 6) confirman, en primer lugar, la posición envidiable de Guadalajara, pero también de la Zona Metropolitana de la Ciudad de México y de otras grandes metrópolis del centro. Es posible suponer que las ventajas del tamańo urbano (economías de aglomeración) y de los factores propios a los clusters industriales prevalecen aquí sobre otras consideraciones. Las ciudades del núcleo histórico de México -el centro- siguen siendo, bajo esta perspectiva, los principales motores de la economía mexicana, con Monterrey como digno rival, a pesar de la atracción indiscutible de la frontera norte. Las ciudades del norte, con la excepción notable de Monterrey y su región, no son grandes centros de servicios superiores por razones que ya se han mencionado. 


\section{Figura 7 \\ CONCENTRACIÓN RELATIVA DEL EMPLEO EN SERVICIOS DE PUBLICIDAD POR REGIÓN Y POR TAMAÑO URBANO (México $=1.00$ )}

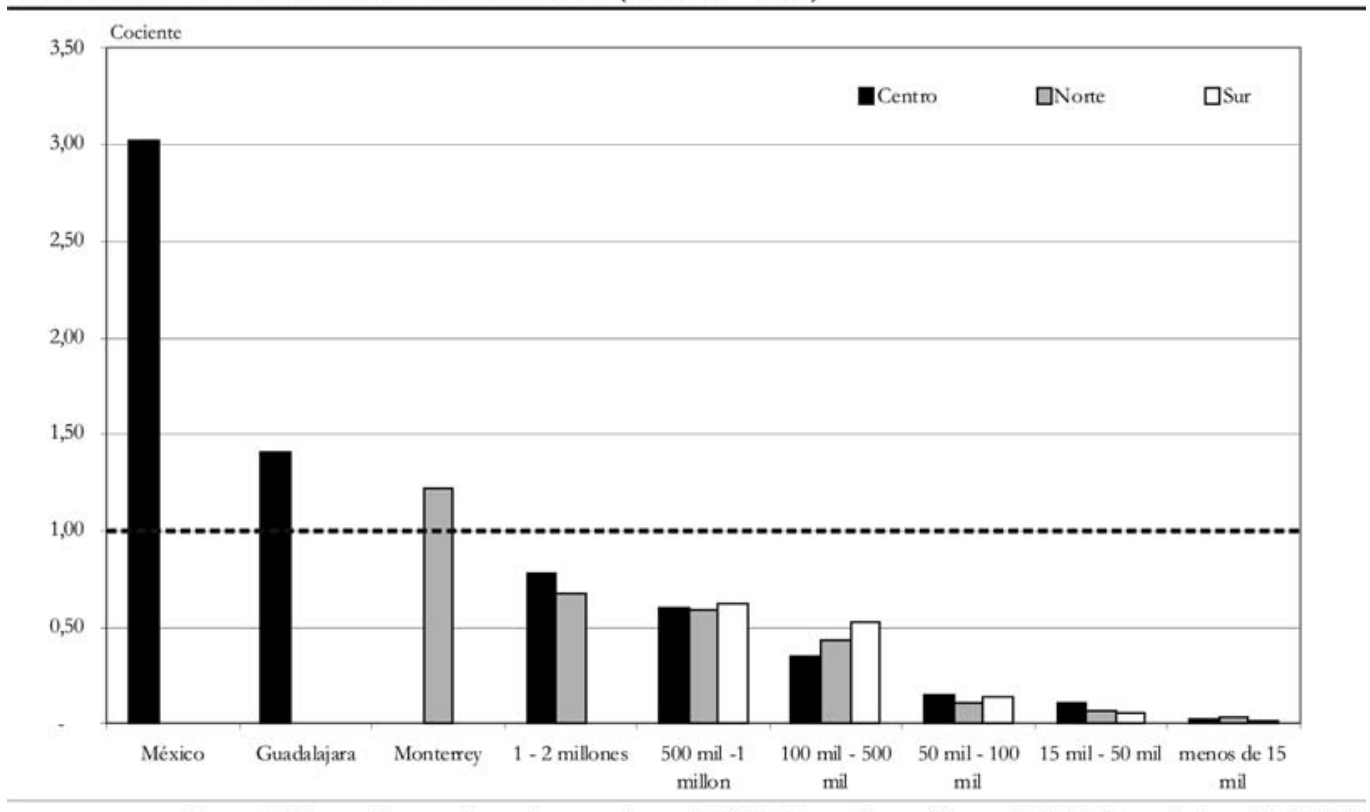

Fuente: Elaboración propia con base en datos del XIII Censo de servicios y del XVI Censo industrial, INEGI

El dominio continuo del centro -y por lo tanto, la importancia de las economías de aglomeración para las actividades modernas- se observa aún más claramente en la Figura 7. La publicidad es uno de los sectores de actividad más sensibles a las economías de aglomeración. Depende de una gran diversidad de talentos y competencias: cantantes, diseñadores, actores, técnicos, músicos, programadores, entre otros. Paralelamente, necesita encuentros frecuentes entre los creadores de publicidad y los directores y demás administradores de las sociedades (o instituciones) que hacen los pedidos, por lo que tiende a acercarse a las grandes sedes sociales o instituciones. La posición dominante de la ZMCM como centro de publicidad ${ }^{24}$ se observa claramente en la figura 7.

\footnotetext{
24 Los mexicanos que ven televisión - casi toda la población mexicana- saben que la publicidad que ven en las pantallas fue, con toda probabilidad, concebida en la capital, y que las personas que aparecen en ellas promocionando tal o cual producto, se encuentran también en la capital. A este nivel, la integración del mercado mexicano, del sur al norte, parece total.
} 


\section{Figura 8 \\ CONCENTRACIÓN RELATIVA DEL EMPLEO EN FINANZAS \\ POR REGIÓN Y POR TAMAÑO URBANO $($ México $=1.00$ )}

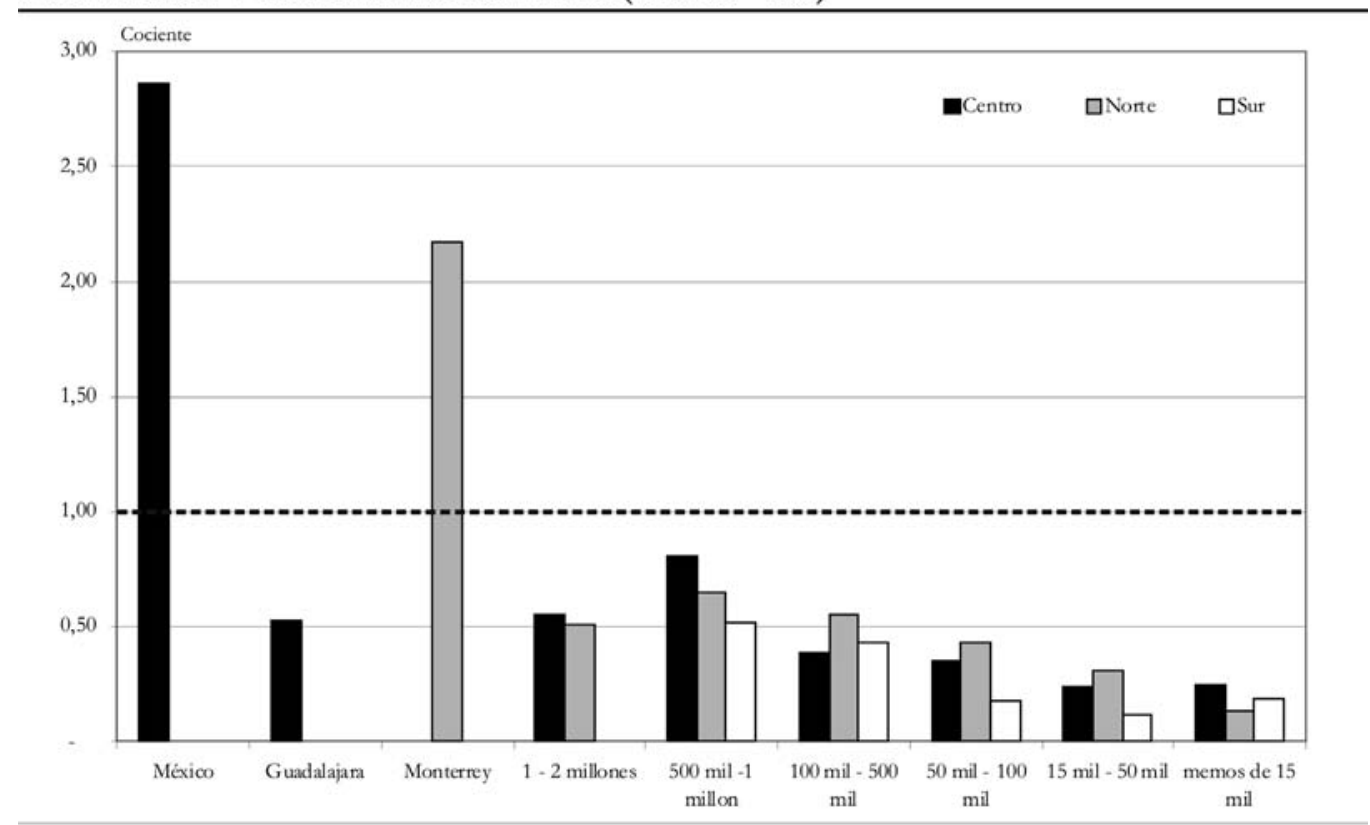

Fuente: Elaboración propia con base en datos del XIII Censo de servicios y del XVI Censo industrial, INEGI

La posición dominante de la capital -con Monterrey como único rival- se observa aún más claramente en los servicios financieros (Figura 8). La fuerte concentración en la Ciudad de México refleja en parte una situación malsana vinculada al subdesarrollo del sector financiero mexicano, particularmente el sector bancario ${ }^{25}$-recién salido de su última crisis-. La mayor parte de las instituciones no tienen la capacidad suficiente para atender a las poblaciones fuera de las grandes ciudades o los destinos turísticos importantes. El resultado es un sector financiero altamente concentrado geográficamente (Garza, 2005; Parr y Budd, 2000). Si retirásemos a Monterrey de la figura, no quedaría más que una ciudad con una concentración relativa superior al promedio nacional: la ZMCM.

\section{Conclusiones}

De acuerdo con nuestros resultados, los patrones de localización de las actividades económicas en México reproducen, en su conjunto, las formas observadas en otros países. La industria manufacturera -sobre todo las industrias de baja y mediana tecnología- no se concentra ya, en términos relativos, únicamente en las grandes aglomeraciones urbanas. Las industrias manufactureras tienden a preferir las ciudades medianas. Sin embargo, la desconcentración

25 En los países en desarrollo, la red bancaria no cubre todo el territorio, es decir, su densidad bancaria (sucursales por kilómetro cuadrado) es muy baja. En México es de 0.004, en Argentina y Chile es de sólo 0.002 y en Brasil y Colombia de 0.003, mientras que en Espańa es de 0.028, 14 veces superior a la de Argentina y Chile, nueve veces la de Brasil y Colombia, y siete la de México (Ruiz, 2004:568). 
industrial sigue siendo reducida en México, y poco presente en las ciudades pequeñas. Por su parte, los servicios superiores, al igual que en otros países, muestran una distribución jerárquica muy sensible al tamaño urbano, y fuertemente concentrada en las metrópolis más grandes del país.

Sería difícil pretender que los factores que dan forma a los patrones de localización sean fundamentalmente diferentes en México. El tamaño urbano (las economías de aglomeración) y la distancia (de los mercados) se destacan como factores determinantes. Sin embargo, la geografía mexicana -especialmente la proximidad con los Estados Unidos y el alejamiento del sur del país- produce modelos distintos de localización. En el norte, haciendo abstracción de Monterrey y su región, buena parte de la industria manufacturera no se localiza en función del criterio de tamaño, sino de la frontera, frecuentemente con respecto a su vis-à-vis urbano norteamericano. El análisis por rama industrial muestra lógicas espaciales distintas, aun dentro de la misma actividad. Así, por ejemplo, la industria del vestido sigue dos lógicas espaciales, dependiendo en qué parte del país se encuentra. Los servicios a las empresas en el norte (nuevamente con la excepción de Monterrey), no obedecen a la misma lógica de tamaño urbano que en el resto del país, porque sus puntos de referencia se encuentran frecuentemente del otro lado de la frontera y no en México.

La lógica espacial del centro se acerca mucho más a lo que podemos denominar el patrón clásico de localización, con la Zona Metropolitana de la Ciudad de México como polo dominante de servicios. El dominio de la capital puede observarse sobre todo en el caso de los servicios superiores como las finanzas y la publicidad, pero también para ciertos servicios más técnicos. A este nivel, la ZMCM sigue siendo el centro de servicios para el conjunto del país, incluyendo el norte. La fuerza de esta metrópolis como centro de servicios superiores la convierte también en el principal punto de expulsión industrial hacia las aglomeraciones vecinas como Puebla y Toluca. Asimismo, se observa en el centro la emergencia de clusters industriales alrededor de Guadalajara para la electrónica. Sin embargo, la debilidad del sector financiero mexicano se traduce en una jerarquía urbana reducida, dominada por un único polo. Únicamente Monterrey se presenta como rival de la capital como centro financiero.

Finalmente, nuestros resultados confirman la posición marginal del sur en lo que se refiere a la localización de actividades económicas modernas. El sur está doblemente desfavorecido por la distancia (del centro y de los Estados Unidos), y por la ausencia de grandes ciudades. El movimiento de desconcentración industrial no ha llegado realmente al sur. Esta región sigue siendo casi un desierto en lo que se refiere al empleo manufacturero. Excepto algunos lugares aislados donde se encuentran los sectores tradicionales como la industria textil y del vestido, la ausencia de una gran ciudad representa un freno al desarrollo de un sector moderno de servicios superiores. Sin gran metrópoli, no es fácil contrarrestar las desventajas relacionadas con la distancia en un país tan vasto y culturalmente diverso como México. Pero esto no es únicamente el resultado de los mecanismos del mercado y sus agentes privados, sino que aboga en favor de una intervención especial. 


\section{Referencias bibliográficas}

Alonso, O.; Chamorro, J. M. \& González, X. (2004). Agglomeration economies in manufacturing industries: the case of spain. Applied Economics, 36, 8, 103-16.

Banco Mundial (2003). Lessons from NAFTA for Latin America and the caribbean countries: a summary of research findings. Washington D.C.: Banco Mundial.

Calderón, C. \& Martínez, G. (2004). Estructura industrial de la frontera norte y estrategia de desarrollo. Comercio Exterior, 54, 8, 712-720.

Castillo, R.; Bautista, A. \& Fragoso, E. (2004). Sincronización entre las economías de México y Estados Unidos: el caso del sector manufacturero. Comercio Exterior, 54, 7, 620-627.

Daniels, P. (1985). Services industries: a geographical appraisal. Methuen, London, New York: Library of Congress, cataloging in publication data.

Delgadillo, J.; Torres, F. \& Gasca, J. (2001). El desarrollo regional de México en el vértice de dos milenios. México: Editorial Miguel Ángel Porrúa e Instituto de Investigaciones Económicas, UNAM.

Desmet, K. \& Fafchamps, M. (2005). Changes in the spatial concentration of employment across US counties: a sectoral análisis (1972-2000). Journal of Economic Geography, 5, 261-284.

Figueroa, L. (2006). Análisis espacial de la captación y el financiamiento bancario en México. En G. Garza (Coord.), La organización espacial del sector servicios en México (pp. 233-274). México: El Colegio de México.

Gaigné, C.; Piguet, V. \& Scmitt, B. (2005). Évolution récente de l'emploi industriel dans les pays territoires ruraux et urbains: une analyse structurelle-géographique sur des données françaises. Revue d'Économie Régionale et Urbaine, 1, 3-30.

Garcia-Verdi, R. (2005). Income, mortality, and literacy distribution dynamics across status in Mexico 1940-2000. Cuadernos de Economía, 42, 125, 165-192.

Garza, G. (1985). El proceso de industrialización en la ciudad de México, 1821-1970. México: El Colegio de México.

Garza, G. (2005). Concentración financiera en la Ciudad de México (1960-2001). EURE, 31, 92. Rescatado el 23 de enero de http://www.scielo.cl/scielo.php?script=sci_arttext\&pid=S0250$71612005009200002 \&$ Ing=es\&nrm=iso.

Garza, G. (Coord.) (2006). La organización espacial del sector servicios en México. México: El Colegio de México.

Gaspar J. \& Glaeser, E. (1998). Information technology and the future of cities. Journal of Urban Economies, 43, 136-56.

Graham, D. \& Spence, N. (1997). Competition for metropolitan resources: the 'crowding out' of London's manufacturing industry. Environment and Planning A, 29, 459-484.

Henderson, V. (1997). Medium sized cities. Regional science and urban economics, 27, 583612.

Henderson, V. (2003). Marshall's scale economies. Journal of Urban Economics, 53, 1-28.

Henderson, V.; Shalizi, Z. \& Venables, A. J. (2001). Geography and development. Journal of Economic Geography, 1, 81-205.

Ingram, G. K. (1998). Patterns of metropolitan development: what have we learned? Urban Studies, 35, 7, 109-1035. 
Klaus, D. \& Fafchamps, M. (2005). Changes in the spatial concentration of employment across US countries: a sectoral analysis (1972-2000). Journal of Economic Geography, 5, 261-284.

Martínez, S. R. \& Rubiera, F. (1999). Identificación y análisis de los patrones regionales de terciarización de la economía espańola. Economía Industrial, 328, 132-45.

Messmacher, M. (2000). Desigualdad regional en México. El efecto del TLCAN y otras reformas estructurales. Documento de Investigación No 2000-4. México: Dirección General de Información Económica del Banco de México.

Moreno, A. \& Escolano, S. (1992). El comercio y los servicios para la producción y el consumo. Madrid: Síntesis.

Ornelas, J. (2004). Impacto de la globalización económica neoliberal en el ordenamiento urbano y territorial. Papeles de Población, 41.

Parr, J. \& Budd, L. (2000). Financial services and the urban system. Environment and Planning, $13,97-118$.

Polèse, M. \& Shearmur, R. (2004). Is distance really dead? Comparing industrial location patterns over time in Canada. International Regional Science Review, 27, 4, 431-457.

Polèse, M. \& Shearmur, R. (2006). Growth and location of economic activity: the spatial dynamics of industries in Canada (1971-2001). Growth and Change, 37, 3, 362-395.

Polèse, M. (1998). Economía urbana y regional: introducción a la relación entre territorio y desarrollo. Cartago: Libro Universitario Regional.

Porter, M. (1998). Clusters and the new economics of competition. Harvard Business Review, $76,6,77-90$.

Porter, M. (2000). Location, competition, and economic development: local clusters in the global economy. Economic Development Quarterly, 14, 1, 15-34.

Rodríguez-Pose, A. \& Sánchez-Reaza, J. (2005). Economic polarization through trade: trade liberalization and regional inequality in Mexico. En R. Kanpur \& V. J. Venables (Comps.), Spatial inequlity and development. Oxford: Oxford University Press.

Rubiera, F. (2005). Los servicios avanzados a las empresas. Dinámicas de localización, patrones de externalización y efectos sobre el desarrollo regional. España: Civitas.

Ruiz, C. (2004). Los desbancarizados: el problema de los mercados financieros segmentados. Comercio exterior, 54, 7, 566-574.

Sassen, S. (1991). The global city. New York, London, Tokio. New Jersey: Princeton University Press.

Silva, A. (2004). La respuesta gubernamental a la falta de acceso a los servicios financieros en México. Comercio exterior, 54, 7, 575-582.

Sobrino, J. (2003). Competitividad de las ciudades de México. México: El Colegio de México.

Stanback, T.; Bearse, M.; Peter J. \& Karasek, R. (1984). Servicios: la nueva economía. México: Publigrafics. 
